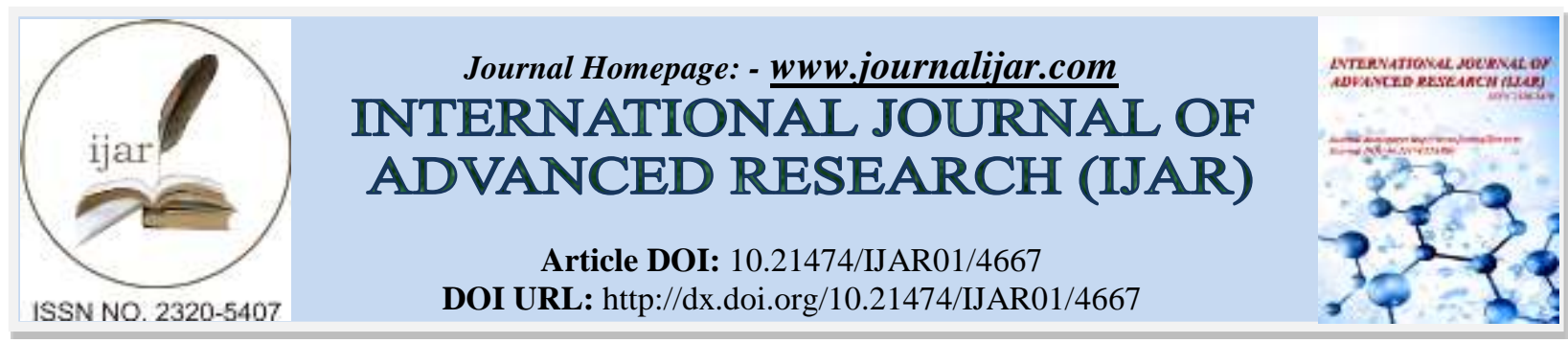

RESEARCH ARTICLE

\title{
SYNERGISTIC ACTION OF STRESS AND ALCOHOL ON THE HIPPOCAMPAL REGION OF BRAIN AND USE OF "Centella asiatica" AS A BRAIN REJUVENATOR.
}

\author{
"Dr. Sachin Gupta and Dr. Anuj Saxena.
}

\section{Manuscript Info}

[..........................

Manuscript History

Received: 28 April 2017

Final Accepted: 30 May 2017

Published: June 2017

\section{Abstract}

In the modern therapeutic system most of the drugs have severe side effects. To overcome this shortcoming use of Ayurvedic drugs are been promoted. We have vast wealth of Ayurvedic, Unani and Siddha systems of medicines, recommending several plants or their extracts having curative or preventive effects against several deformities. Now a days several plants are used as diuretic, sedatives, anti-tumor, antiulcer, anti-stress, skin-modifiers, appetite-increaser, etc. In present study Centella asiatica, which is commonly known as "Mandukaparni", has been used as neuro-protector against the neural degeneration with special reference to Hippocampus."Hippocampus" is the part of brain known to carry out functions like learning, transforming information, discrimination,etc. The activity in the Hippocampus is disrupted by stress and alcohol intake, interacting directly with Hippocampal neurons. In present study Centella asiatica has been used as neuroprotector, which is commonly known as "Mandukaparni".Present study special emphasis has been laid to see the histo-pathology of Hippocampal sub-regions, created due to stress and alcohol intake on albino rats and efforts have been made to study neural degenerations in CA1, CA2, CA3, CA4 and Dg sub-regions of Hippocampus. Here effectiveness of drug has been shown on learning and memory and it is classified as Medhya rasayan.Our results reveal Centella asiatica as neuro-protective agent; its use with stress and alcohol showed least degeneration in different sub-regions of Hippocampus.

Copy Right, IJAR, 2017,. All rights reserved.

\section{Introduction:-}

Brain is the chief controller of our body. It is made of three main parts: forebrain, midbrain, and the hindbrain. Limbic system is situated in the forebrain. The major structures in this system are the mamillary body, the septum (or septal area), the Hippocampus, the amygdala and lingulate cortex.

The Hippocampus is a very large area in the limbic; with an overall curving thus was named as "Hippocampus". It is the part of brain known to carry out functions like learning, transforming information, discrimination,etc. Studies also shows the importance of Hippocampus in spatial memory, the ability to remember where an animal has been and where critical events have occurred (Morris et al; O' Keefe and Conway).

A cross-section taken perpendicular to the long axis (septal-temporal) will reveal the internal structure as two interlocking "Cs", one reversed in relation to the other, each with its own principal cell layer. One "C" makes up 
Ammon's Horn or Cornu Ammonis (CA1 -CA3), also known as the "Hippocampus proper". The principle cell layer of Ammon's Horn is the stratum pyramidale, or the pyramidal cell layer. The other " $\mathrm{C}$ " is made up of the Dentate Gyrus, of which the stratum granulosum, or granule cell layer is the principal cell layer. Although the Dentate Gyrus is commonly included as part of the Hippocampus, it is cyto-architectonically distinct from the Hippocampus proper (the architecture, or structure of the cells are different. However, sometimes the Hilus of the Dentate Gyrus, the area inside the "C" created by the granule cells is referred to as CA4.In recent years, some of the cellular and molecular mechanism which are involved in learning and memory have been extensively investigated in Hippocampus.

The primary area of the brain that deals with stress is its limbic system. Because of its enormous influence on emotions and memory, the limbic system is often referred to as the "emotional brain. "Stress" may be defined as the pattern of bodily responses to any challenge as an event, which precipitates various body changes or as the complex interaction that occur between stimuli, target organism and adaptive responses. Situations, which require adjustment, can be stressful.

Cohen et al (1993) investigated three aspects of psychological stress viz., negative effect, perceived stress and stressor exposure. These three aspects of psychological stress can be seen as providing information about the extent to which an individual feels equipped to handle the stressor to which $\mathrm{s}(\mathrm{he})$ is exposed, and also provide information about stress perception and stressor exposure. Majority of previous studies suggest that it is the stress perception, and not stressor exposure, which predict negative health symptoms (Matthews et al, 1996).

Alcohol besides affecting other body parts has most detrimental effect on brain. It significantly lowers performance on cognitive ability tasks (Pickworth et al) such as problem solving. More complex the task, the more is the organic impairment, including brain shrinkage, observed in a high proportion of alcoholics (Errico et aL), especially among binge drinkers, people who abuse alcohol following periods of sobriety (Hunt). Studies show that most problem drinkers are men, with about five times the frequency of women (Helzer et al).

Alcohol has complex and seemingly contradictory effects on the brain. At lower level, alcohol stimulate certain brain cells and activates the brain's "pleasure areas", which release opium like endogenous opioids that are stored in body (Van Ree).

At higher levels alcohol depresses brain functioning, inhibiting one of the brain's excitatory neurotransmitters, glutamate, which in turn slows down activity in parts of the brain. Inhibition of glutamate in the brain impairs the organism ability to learn and affects the higher brain centers, impairing judgment and other rational process and lowering self-control. As behavioral restraints decline, a drinker may indulge in the satisfaction of impulses ordinarily held in check. Some degree of motor dis-coordination soon becomes apparent and the drinker's discrimination and perception of cold, pain and other discomforts are dulled.

The activity in the Hippocampus is disrupted by stress and alcohol intake, interacting directly with Hippocampal neurons.Normally it is observed that consumption of alcohol is a result of physical or mental stress and alcohol is taken as relaxant. Efforts have been made in present study to prove detrimental effect of stress and alcohol in different sub-regions of Hippocampus.

We have vast wealth of Ayurvedic, Unani and Siddha systems of medicines, recommending several plants or their extracts having curative or preventive effects against several deformities. Now a days in Ayurvedic system several plants are used to overcome the side effects in modern therapeutic system.

Branch of Ayurveda known as "Medhya Rasayan" constitute several herbal drugs like Brahmi, Shankhpushpi, Jatamasi, Mandukaparni, etc. which are used as brain rejuvenators. Extracts of these plants have their affect directly on the brain, improving the short-term and long-term memory.

In present study Centella asiatica has been used as neuro-protector, which is commonly known as "Mandukaparni".

"Mandukaparni" is sour and sharp in taste, easily digestible, having cold effect, nutritive and fever curer. It is also useful in bronchitis, inflammation and asthma. It improves memory, diet and voice. It also improves the blood vessels inside the skin so; it is very helpful in diseases related to skin and urinary system. Indian practitioners are using "Mandukaparni" to cure various skin diseases. 
In this study we have planned to see the effect of stress and alcohol after their chronic exposure and preventive and repairable effects of Centella asatica in the various sub-regions of Hippocampus and to observe changes in behaviour of animal after intake of drug under experimental conditions and protocol.

The present study has been taken to project and take up further research for establishing neuro-protective nature of "Mandukparni" against the neural degeneration with special reference to Hippocampus. Aspect was chosen keeping in mind the fact that, a vast potential of medicinal plants are lying untapped having very positive clinical results with least side effects and we still lack a serious effort to promote Medhaya rasayana as a safe and effective alternative to existing synthetically manufactured organic drugs for treating the neural degeneration.

In the present study special emphasis has been laid to see the histo-pathology of Hippocampal sub-regions, created due alcohol intake, immobilization stress and their synergistic effects on albino rats. Efforts have been made to correlate the exhibited neural degenerations in CA1, CA2, CA3, CA4 and Dg sub-regions of Hippocampus with behavioral changes.

In recent years, some of the cellular and molecular mechanism which are involved in learning and memory have been extensively investigated in Hippocampus.

\section{Material And Method:- \\ Experimental Animal:- \\ Control Group:-}

Cages with rats were kept in isolated room and locked for 24 hours to avoid the stressful situation due to handling. Body weight, food intake and water intake of entire group was recorded both prior to the start of an experiment (7 days) and daily during the experiment period (30 days). The animals were decapitated immediately after taking out from cage and brain was dissected, trimmed and fixed in $10 \%$ neural formaldehyde at $4^{\circ} \mathrm{C}$ for 16 hours.

\section{Experimental Group:-}

\section{Alcohol + Stress + Drug}

For studying neural degeneration in Hippocampal sub-region in this group animals were given herbal drug, after subjecting them to stress + alcohol protocol.

1. 40 animals were taken for study.

2. Similar set of 10 animals were decapitated on 1st day after one-day treatment of stress, alcohol and herbal drug. Sections were prepared on cryostat stained with crystal violet stain for observing sub-region.

3. Another set of ten animals were continuously given stress, alcohol and drug for 15 days and were decapitated on 15 th day to prepare normal histological slides.

4. Ten animals were decapitated on 30th day of treatment with stress, alcohol and drug. Normal section were cut on rotary cryostat and stained with crystal violet to observe changes.

5. Rest ten animals, kept for control decapitated on 30th day and histological sections were prepared as permanent slides after crystal violet staining.

\section{Alcohol Protocol:-}

Pearl pointed syringe was used for administration of marked dose ( $3 \mathrm{gm} / \mathrm{Kg}$ of body weight) of alcohol to the animal (West et al, 1985). To give alcohol, albino rats were hold vertically with mouth placed upside and then alcohol is pushed through gullet into the body with the help of syringe. In the experimental groups alcohol was administered after stress protocol.

\section{Stress Protocol:-}

Immobilization stress was given to the rats. Rats were kept individually in tight and ventilated plastic boxes for 2 hours/day for number of days as per protocol. Rats were fitted so tightly that they were unable to move in the box and hence got stressed.

\section{Drug Protocol:-}

The marked dose of drug (400mg/kg of body weight) was administered to the animal orally with the help of pearl pointed syringe. The animal was hold vertically upside with mouth placed upside and drug was pushed to the body through the gullet. In this experiment drug was always administered after stress and alcohol protocol. 


\section{Histological Study:-}

The rats were sacrificed by decapitation after cervical dislocation without any anesthesia, brain was dissected out immediately, after washing with distilled water, tissues were fixed in $10 \%$ natural chilled formaline or bouin's fixative for $16 \mathrm{hr}$. at $4^{\circ} \mathrm{C}$. Sections of $10 \mu \mathrm{m}$ thickness were cut on rotatory microtome and studied.

\section{Observation:-}

\section{Histology:-}

The experimental groups including namely control(1), stress only (2), Alcohol only (3), Alcohol + Stress (4), Alcohol + Stress + Drug (5), Drug only(6) were studied. After subjecting the animals to these conditions they were decapitated and histological slides of their brains were prepared to expose the CA1, CA2, CA3, CA4 \& DG region of their hippocampus. Neuron cell bodies of these sub-regions were observed under magnification (X40) of microscope. Identification of Hippocampal sub-regions was based on "Rat Brain" in stereotoxic coordinates atlas (Paxinos and Watson, 1982).

Following results were observed in different experimental groups:

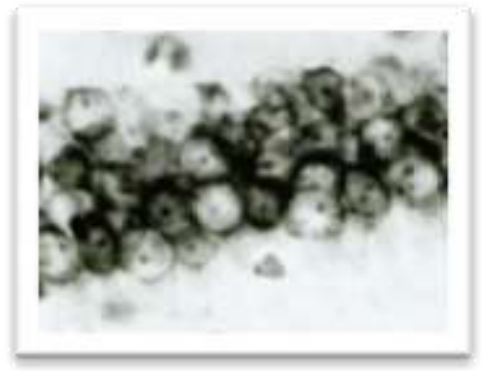

Slide 1:- Control group- Healthy neurons with clear nuclei.

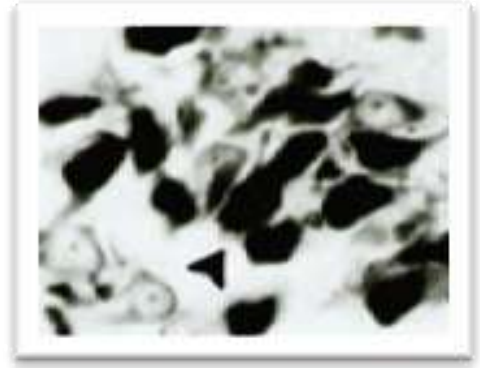

Slide 2:- Stress group- Damaged pyknotic neurons.

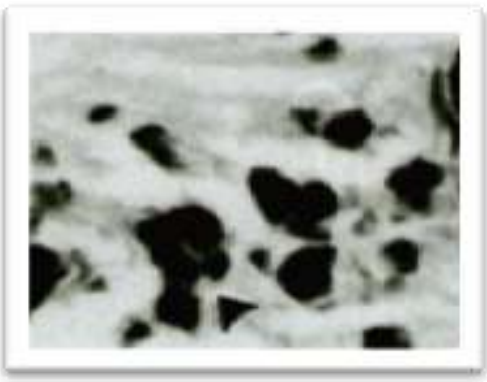

Slide 3:- Alcohol only- Damaged pyknotic neurons.

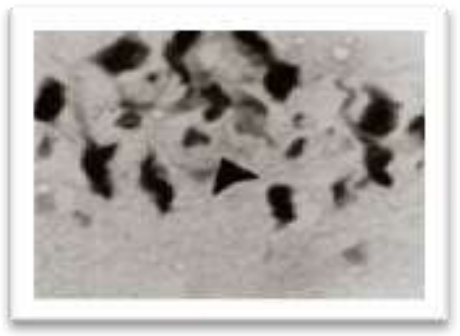


Slide 4:- Alcohol + Stress- Damaged pyknotic neurons.

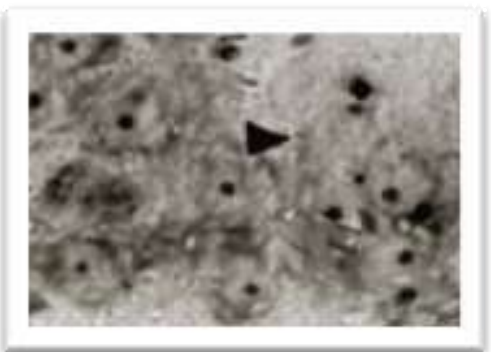

Slide 5:- Alcohol + Stress + Drug- Recovering neurons.

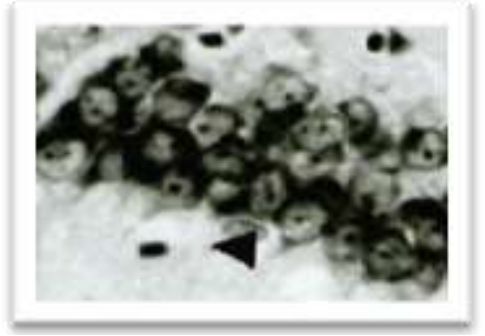

Slide 6:- Drug only- Healthy neurons with clear nuclei.

\section{Result And Discussion:-}

The role of hippocampus in memory, learning, discrimination and behavioral activities has now been established.

Stress is a condition or stimulus that threatens an organism's survival (Chrousous and Gold). Stress can be beneficial or detrimental to the survival of an organism, leading to behavioral or physiological changes (Herman and Cullinan,).

This stress system has a close integration with other central nervous system and the elements involved in the regulation of behavior and emotion in addition to axis responsible for growth, immunity and reproduction (Abraham and Gogate, and Baum et al). The brain sub-regions has been found to be directly affected by stress expressed in the form of varying degree of cellular degeneration resulting into impaired memory. Stress, thus has been found to be directly involved in causing Hippocampal degeneration.

The result of present study demonstrated that one month of immobilization stress caused significant degeneration in Hippocampal cells which were seen in form of pyknotic, condensed and shrunken cells lacking cytoplasm.

The consistency of our result was not found with the results given by Uno, who did not reported any loss of pyramidal cells in Hippocampal region of female monkey. Another, contrary finding was given by Harding et al where it was reported that changes observed in rodent model of alcoholism were not found parallel to those observed in the humans.

Fuch and Flugg confirmed degeneration of Hippocampal sub-region due to chronic stress causing dendritic atrophy of Hippocampal pyramidal neurons due to elevated level of Glucocorticoid Another probable reason, which explains Hippocampal damage due to prolonged immobilization stress, may be due to the fact that stress regulates neurotrophic factor expression in the brain. Stress has been found to decrease brain derived neurotrophic factor (BDNF), mRNA in Hippocampus leading to stress induced damage (Smith). Nerve growth factors (NGF) and neurotrophin 3 (NT3) get increased by stress and Glucocorticoid perhaps as a compensatory response to stress induces damage.

Neurotrophic factors being neuro-protective in nature may also be effective in reversing Glucocorticoid-induced damage to Hippocampus. These factors plays very important role in protecting the brain from variety of traumatic insult. The advance research in the field of neuroscience suggest that oxidative stress may also play an important role in neuron loss in neuronal degenerative diseases such as Parkinson and Alzheimer's disease. Oxidative stress is 
a cyto-pathological condition caused due to mismatch between free radical production and ability of cell to define against them (Simonian).

However, alcohol related neuronal loss has been found in specific region of the cerebral cortex (superior frontal association cortex) and hypothalamus (supra optic and paraventrical nuclei). But no change has been reported in basal ganglia nucleus basalis or serotonergic raphe nuclei. The functional changes and cognitive deficits in uncomplicated alcoholics have been documented due to dendritic and synaptic changes together with changes in reception and transmission that precedes the more severe structural neuronal changes. Neuronal loss in hypothalamus and cerebellum causes pathological changes that have been found to correlate with alcohol intake (Harper and West et al).

More vulnerability of mature neurons to ethanol induced hypoxic episodes may be accounted for neural degeneration cause by alcohol. The probable reason for this may be high dependence on oxidative phosphorylation and use of ATP. Mature cells may also be more sensitive to ethanol-induced disruption of intracellular $\mathrm{Ca}++$ (Michaelis and Michaelis).

Newlin, in 1981 evaluated effect of ethanol on synaptic transmission in rat Hippocampus, since the action of psychoactive drug effect the neuronal activity. Indeed the systemic application of ethanol at doses associated with behavioral intoxication increase the excitatory and inhibitory responses of pyramidal cell to stimulation of different pathway. The systemic does of ethanol selectively potentiates excitatory responses to acetylcholine(Ach) and inhibitory responses to somatostanin-14(SS-14)but do not affect responses to other neurotransmitters in Hippocampus.

Therefore, in the recent times, there has been a massive hunt for all such herbal formulations, which are effective against neural disorders. Centella asiatica has been used as neuro-protector against synergistic action of alcohol and drug with the same intension. The drug was found to be effective in preventing further neural degeneration and thus controlling damage to Hippocampal cells caused by action of alcohol. Hippocampal sections of experimental animals after treatment with drug shows least neural degeneration in CA1, CA2, CA3, CA4 and Dg region thus exhibiting the neuro-protective nature of drug.

Extract obtained by Centella asiatica is commonly used in the Ayurvedic system of medicine to treat various diseases. Our study gained support from the work of (Babu, Kuttan and Padikkala), they reported that crude extract and partially purified fractions of Centella asiatica has anti-tumer effect in mice.

Centella asiatica shows improvement in the central nervous system, as its asiaticoside derivatives were found to inhibit or reduce $\mathrm{H} 2 \mathrm{O} 2$ induced cell death and lower intracellular free radical concentration, protecting against the effect of $\beta$ amyloid neurotoxicity (Mook Junk et al,). Our study for using Centella asiatica as neuroprotective drug is supported by the work done by Leung and Foster. They studied the assessment of turnover of biogenic amines (norepinephrine, dopamine and serotonin) and showed significant reductions of these amines and their metabolites in the brain following administration of fresh juice. The decrease in amine levels was correlated to improved learning and memory in rats.

Leung and Foster, observed that a water-soluble fraction of Centella asiatica was found to have an anxiolytic effect in animals comparable to diazepam. The extract of Centella asiatica was found to increase brain GABA levels (Chatterjee at al,).

Heinerman, observed that Mandukaparni is exceptionally high in B Complex vitamins, especially B1, B2 and B6 all of which are essential for the correct function of the nervous system.

Our observations got due support with results of (Jaiswal \& Bhattacharya), where effectiveness of drug has been shown on learning and memory. Categorizing it as a nootropic agent and classifying it as Medhya rasayan.

Our results reveal Centella asiatica as neuro-protective agent; it's use with alcohol showed least degeneration in different sub-regions of Hippocampus.

Neural degeneration caused due to stress may be checked with herbal drug categorized under Medhya Rasayana. In present work Centella asiatica has been taken as neuro-protector. 
The use of Centella asiatica as a neuro-protector has opened a new field to control the adverse effect of alcohol and stress on the brain. Improvement as shown by Centella asiatica for enhancing memory and intelligence needs further studies to control and recover neural degeneration caused by stress, alcohol intake and other day to day factors.

\section{Acknowledgement:-}

The authors are thankful to the Head of the Department of Zoology, University of Rajasthan, Jaipur and Department of Zoology, Govt. R.R.(PG) Autonomous College, Alwar (Raj.) for providing laboratory facilities.

\section{References:-}

1. Abbott, D. H. (1987). Behaviorally mediated suppression of reproduction in females pumates. J. Zool. (Lond.) 213:455-470.

2. Abraham, M.E. and Gogate, M.G. (1989). Effect of stress on Behaviour in rats. Ind. J. Physiol. Pharmac. 33(2) : 84-89.

3. Canon, W. B. (1914). The James-Lange theory of emotions: A critical examination and an alternative theory. American Journal of Psychology, 39, 106-124.

4. Cohen, C. A., Gold, D. P., Shulman, K. L., \& Wortley, J. T. (1993). Factors determining the decision to stitutionalize dementing individuals: A prospective study. Gerontologist, 33(6), 714-20.

5. Fuchs, E. and Flugg, G. (1998). Stress, Glucocorticoirds and structural plasticity of the hippocampus. Neuro science and Beh. Review 23(2): 295-300

6. Gluck, M. and Myer, C. (1993). Hippocampal mediation of stimulus representation. A computational theory. Hippocampus. 3: 491-576.

7. Harding, A.J., Wong, A., Sroboda, M., Kril J.J. and Halliday, G.M.(1997).Chronic alcohol consumption does not cause hippocampal neuron loss in humans : Hippocampus : 7 (1) : 78-87.

8. Herman and Cullinan. (1997). Neurocircuitry of stress: central control of the hypothalamo-pituitaryadrenocortical axis. Trends in Neurosciences 20 (1997) 78-84.

9. Simonian, N.A. and Coyle, J.T. (1996). Oxidative stress in neurodegeneration diseases. Annual review of pharmacology and Toxicology .36:83-106.

10. Simonov, P.V. (1974). On the role of the hippocampus in the integrative activity of brain. Acta Neurobiol Exp. 24: 33-41.

11. Smith, M.A. (1996) Hippocampal vulnerability to stress and aging: possible role of neurotrophic factors. Behav Brain Res. 78(1): 25-36.

12. Uno, H., Else , J.G., Suleman. M.A. and Sapolsky , R.M. (1989) . Hippocampal damage associated with prolonged and fetal stress in primates J Neurosci. 1705-1711.

13. Babu, T.D.,. Kuttan, G. and. Padikkala , J.(1995). Cytotoxic and anti-tumour properties of certain taxa of Umbelliferae with special reference to Centella asiatica (L.) Urban. J Ethnopharmacol. 48(1):53-7

14. Errico, A. L., Parsons, O. A., \& King, A. C. (1991). Assessment of verb sequential and visuopatial cognitive abilities in chronic alcoholics. Psychol. Asess. 3, 693-96.

15. Harper, C. (1998). The neuropathology oif alcohol - specific brain damage or doses alcohol damage the brain?. Journal of neuropathology and experiment neurology. 57 (2) : 101-10.

16. Heinerman, J. (1984a). An Herb for Our Time: The Scientific Rediscovery of Gotu Kola. Unpublished paper.

17. Helzer, J. E., Canino, G. J., Yeh, E. K., Bland, R., et al. (1990). Alcoholism - North America and Asia: A comparison of population surveys with the Diagnostic. Interview Schedule. Arch. Gen. Psychiat., 47(4), 313-19.

18. Hunt, W. A. (1993). Are binge drinkers more at risk of developing brain damage? Alcohol 10: 559-61.

19. Jaiswal, A.K. and Bhattacharya, S.K. (1992). Effect of Shilajit on memory anxiety and Brain monoamines in rat. Indian j. of pharmacology; (24): 12-17.

20. Leung, A.Y. and Steven Foster. (1996). Encyclopedia of Common Natural Ingredients used in food, drugs and cosmetics. 2nd. ed. New York-Chistester: John Wiley.

21. Maher, J. J. (1997). Exploring effects on liver function. Alcohol Health \& Research, 2(I), 5-12.

22. Michaelis, E.K. and Michaelis, M.L. (1986). Molecular events underlying the effect of ethanol on the developing nervous system in J.R. West (Ed.). alcohol and brain development. New Youk : Oxford Univ. Press: 277-309.

23. Mook-Jung, I., Shin,J.E., Yun, S.H., Huh, K, Koh, J.Y., Park, H.K, Jew, S.S. and Jung, M.W. (1999). Protective effects of asiaticoside derivatives against beta-amyloid neurotoxicity. J Neurosci Res. 58(3):417-25

24. Morris, R. G., Garrud, P., Rawlins, J. N. P. and O'Keefe, J.(1982). Place navigation impaired in rats with hippocampal lesions. Nature (London). 297: 681-683. 
25. Newlin, S.A., J. Mancellas - Trerino, and Bloom, F.K. (1981). Effect of ethanol on behavioural activity of albino: a comprehensive study . brain Res. 209,113.

26. Pickworth, W. B., Rohrer, M. S., \& Frant, R. V. (1997). Effects of abused drugs on psychomotor performance. Experimental and Clinical Psychopharmacology, 5(3), 235-41.

27. Van Ree, J.M.(1996). Endorphins and experimental addiction. Alcohol. 13(1):25-30. 